\title{
An Advance Approach to Select Initial Seed Pixel using Edge Detection
}

\author{
Rajesh Gothwal \\ M. Tech. [CS] \\ Department of Computer \\ Engineering \\ Govt. Engineering College \\ Ajmer, India
}

\author{
Deepak Gupta \\ Assistant Professor \\ Department of Computer \\ Engineering \\ Govt. Engineering College \\ Ajmer, India
}

\author{
Shikha Gupta \\ Assistant Professor \\ Department of Information \\ Technology \\ Govt. Engineering College \\ Ajmer, India
}

\begin{abstract}
This paper proposes a method of initial seed pixel selection used for image segmentation based on edge detection technique. Initial pixel seed selection is the crucial and starting stage of image segmentation. This method is based on RGB color model. In this paper author use the gradient magnitude of the Red, Green and Blue components of true color image. Two type of information are used: non-edge information and similarity behavior of pixels to its neighbors. Edge detection technique is used to select initial seed pixel selection by computing gradient of the image intensity function. The Gradient magnitude provides image with regions having high intensity variation. The threshold value is used to obtain seed pixels and its value depends on the nature of the image.

In this paper the comparative analysis of various images with different Edge Detection techniques is presented. The analysis of images with different edge detection techniques is presented using image processing tool MATLAB 7.5.0. It has been shown that gradient based edge detection techniques provides similar and better result than other edge detection techniques.
\end{abstract}

\section{Keywords}

Image, segmentation, seed selection, non-edge information, threshold value.

\section{INTRODUCTION}

Image processing is a technique used to obtain an enhanced image that is more useful or pleasing to human observer or to perform source of interpretation and recognition tasks usually performed by human for various applications. In last few decades many image processing techniques have come into existence. Mostly techniques are developed to improve the quality of the images that are obtained from spacecraft, space probes and military reconnaissance. Image processing tools have become popular due to easy availability of personnel computers, low cost and processing software.

Digital image processing involves the activities ranging from capturing image with the use of the camera then processing the image to improve quality and to extract the useful information from image. These information are then used in various fields of technology. The main purpose of digital image processing is to allow human beings to obtain high quality image with descriptive features of the original image. Digital Image processing is the technology of applying a number of computer algorithms and computations to process a digital image. The output of the process can be an image or set of descriptive properties or characteristics of the original image. Digital image processing directly deals with the image which is composed of pixels. These pixels are of spatial coordinates that indicate the position of the pixels in the image and intensity values in the gray scale image.

Image segmentation is one of the most important techniques for image processing-based applications and affects the overall image processing system. Segmentation is process of extracting interesting objects for further processing such as description and recognition. It has application in several domains like Medical Science, Analysis of Remotely Sensed Image, Finger Print Recognition and Traffic System Monitoring and so on. Image segmentation in case of the gray scale image sub dividing a given image into its constituent objects based on the intensity and in case of color image segmentation based on the colors with other properties such as texture. Image segmentation means division of an image into meaningful structure. It is the process of extraction and representing information from the image to group pixels together with region of similarity. In the seed based color image segmentation method selection of initial seed is crucial step because it decides the overall segmentation by region growing technique.

\section{A BRIEF OVERVIEW OF THE RELATED WORK}

The selection of seed pixel is the crucial step in the color image segmentation techniques. There is no standard approach for seed pixel selection. When seed pixels are selected then these pixel acts as pivot. These pixels are used in region growing techniques. The region growing techniques are based on the similarity between the pixels. If the seed pixel and neighboring pixel have similar property then seed pixel and neighboring pixel should reside in a single region. Seed selection affects the overall technique of color image segmentation. The pixels selected as seeds known as initial seed pixel should not reside on edges and have similar property of pixels to its neighbors. Non-edge means the pixels are not on the edges. Edges are the part of the images where intensity changes abruptly, so seed pixel should not reside at edges. The meaning of similarity means the seed pixel and its neighboring should have similar property and they belong to same region. The idea of seed pixel for region growing in image segmentation had given first time by Rolf Adams et al. [1]. The proposed method did 
not use any particular procedure to select initial seed pixel for seeded region growing. The method takes a set of pixels as seed pixel. Obviously this method has less probability to provide good result as no criteria is used for seed pixel selection

An effort made by Om Prakash Verma et al. [2] towards reduction in computational cost and execution time in image segmentation by a proposed method. This method does not use any procedure to select seed pixel for region growing. In this method the center pixel of the image is selected as the initial seed pixel. The seed pixel grows region according to grow formula and select next seed from connected pixels of the region. The problem with this method is that there is only one seed pixel so this method has complex process for color image segmentation. An approach proposed by Frank Y. Sihih et al. [3] first time for seed pixel section in image segmentation. The technique defined three criteria: First, Seed pixel must have high similarity to its neighbors. Second, for an expected region at least one seed must be generated in order to produce this region. Third, seeds for different regions must be disconnected.

Biplab Banerjee et al. [4] used Minimum spanning tree (MST) data structure for color image segmentation. The proposed technique first perform (MST) based "natural grouping" of pixel. The MST based natural grouping is used to find the clusters of pixels having similar RGB values. The pixel that is nearest to the center of cluster marked as seed and used for region growing based image segmentation. CIE $\mathrm{L} * \mathrm{a} * \mathrm{~b}$ color space based technique proposed by Gracia Ugariza et al. [5] proposed a technique of seed selection. This technique use CIE $L * a * b$ color space to exploit the information obtained from detecting edge in color image. This technique uses color gradient detection technique to cluster pixels without edges. HSV color model for color image segmentation has used by A. V. Anjikar et al. [6]. This technique uses HSV color model for seeds. Seed selection technique uses two type of information: one is non-edge pixels and second is smoothness feature of the pixels. Noneedge information used to obtain the pixels that are not on the edges and smoothness provides pixels have similar property that help to form a region of the entire pixel have similar properties.

This paper proposed a technique of initial seed selection. This technique uses two types of information non-edge information and similarity behavior. None edge information provides those pixels that are not the edges and similarity behavior help to form a region of pixels having similar properties.

\section{PROPOSED WORK}

The proposed method uses RBG color model and gradient magnitude features of the image processing. The complete method can be described in four phases:

\subsection{RGB Color Model}

RGB color model is a common color model. This color model is a linear color model in which red, green and blue light are added together to produce large number of colors. RGB color models are used in TV and computers to display images. RGB color model is perceptually nonlinear. The reason of use RGB color model for initial seed selection because it is subset of colors that human can perceive. The Red, Green and Blue component are extracted from the input color image that are used in the next phase.

\subsection{Edge Detection}

Edge detection is an image processing technique which determines sharp discontinuity in an image. Sharp discontinuities are rapid change in pixel intensity which shows edge and line in an image [12] [13]. The result of operation of edge detection is an image shows large gradient to abrupt changes in an image while returning values of zero in uniform regions. There are mainly two types of edge detection techniques: Gradient Based Edge Detection and Laplacian Based Edge Detection. Gradient based edge detection techniques deals with the maximum and minimum in first derivative of image i.e. sobel, prewitt etc. Laplacian based edge detection based method finds abrupt intensity change areas in image by searching for zero crossing in second derivative of image.

\subsection{Gradient Magnitude}

The gradient magnitude of the image is extensively used in image processing to help in the obtaining of object contours and separation of homogenous regions [9]. The sobel operator is first order edge detection operator used for obtaining the gradient of the image. The gradient of image provides change of intensity and direction of the change, as sobel operator calculates not only magnitude of the edge but also direction of magnitude. A gradient magnitude of the input image provides the edges at which pixel change their intensity values abruptly. We obtained the gradient magnitude of three components Red, Green and Blue that shows edges and similar area in each component.

\subsection{Threshold Value}

The threshold value is a point beyond which some result begins to produce. In the above step we have obtained combined gradient of three components. Now we obtain gray scale image with use of output image. In gray scale image each pixel has intensity value. We obtain binary image on the basis of threshold value $(\Delta)$ with the use of gray scale image. The pixels in binary are initial seed pixels for image segmentation. Seed pixels are shown in image with red color. The threshold value depends on the nature of the image. We try different threshold values for best result.

The initial seed selection algorithm is described as following steps:

1) Three components Red, Green and Blue are obtained from the input color image.

2) Gradient magnitude of the above three component are obtained using 'sobel' edge detection operator.

3) Gradient magnitude of three components is added to provide a single color image. In this color image pixels that have similar properties belong to a particular region.

4) The proposed technique use threshold value $(\Delta)$ to obtain initial seed pixel. The value of threshold depends on the nature of the color image.

The complete process of proposed technique is also shown in figure 1 . The technique starts with color image as input. The color image contains Red, Green and Blue components. These components show the all color of image when mixed in proper ratio. All these components are extracted and gradient magnitude of each component are calculated using sobel edge detection technique. The obtained gradient 
magnitude of each component combined together that forms RGB color image. The resultant image now converted into gray image. After that the threshold value is used to obtain seed pixel from the gray value. The value of the threshold depends on the objective and type of image and its range is from 0 to 255. If the threshold value is low it provides good results but it makes image segmentation process difficult and lengthy. On the other side if the threshold selected is high, large number of seed pixels is selected but have a little possibility of incorrect result. In this paper threshold value $(\Delta)=100$ is used. In final step seed pixels obtained in binary image are shown in original color image with red color. Figure 2 shows the results obtained with

proposed technique with an image obtained from Berkeley image segmentation dataset.

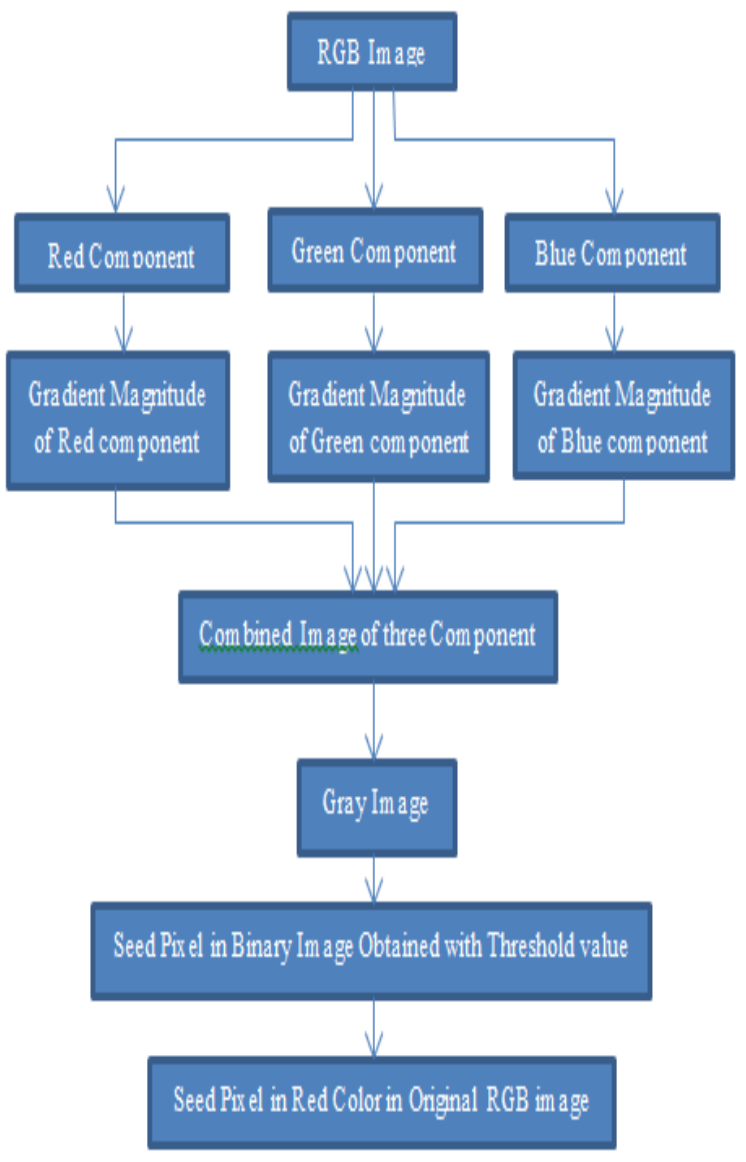

Figure 1: Proposed technique for initial seed pixel selection.

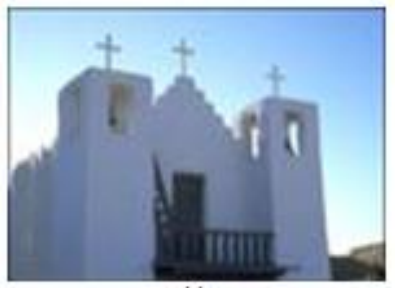

(a)

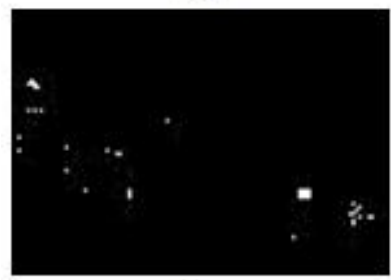

(c)

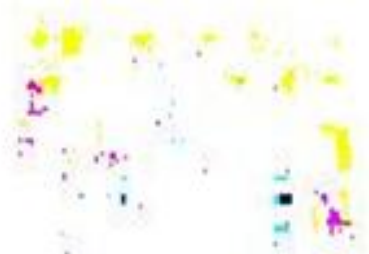

(b)

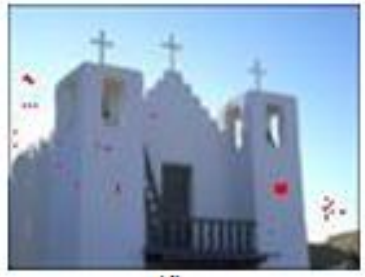

(d)
Figure 2: Initial seed pixel selection using sobel edge detection technique (a) RGB color Image (b) Combined gradient image of Red, Green and Blue component (c) Seed pixel in binary image with threshold value $(\Delta)=100$ (d) Seed pixel in red color in original RGB image.

\section{PERFORMANCE ANALYSIS}

A large number of edge detection techniques exist. In this paper the author consider the sobel, prewitt and laplacian edge detection technique. Sobel and prewitt edge detection are based on gradient magnitude of image that is result of first derivative of the image. Laplacian is an edge dection technique based on second derivative of an image. Figure 3 shows that seed pixels of five images with sobel, prewitt and laplacian edge detection technique. The proposed technique is applied to all five image with threshold value $(\Delta)=100$.

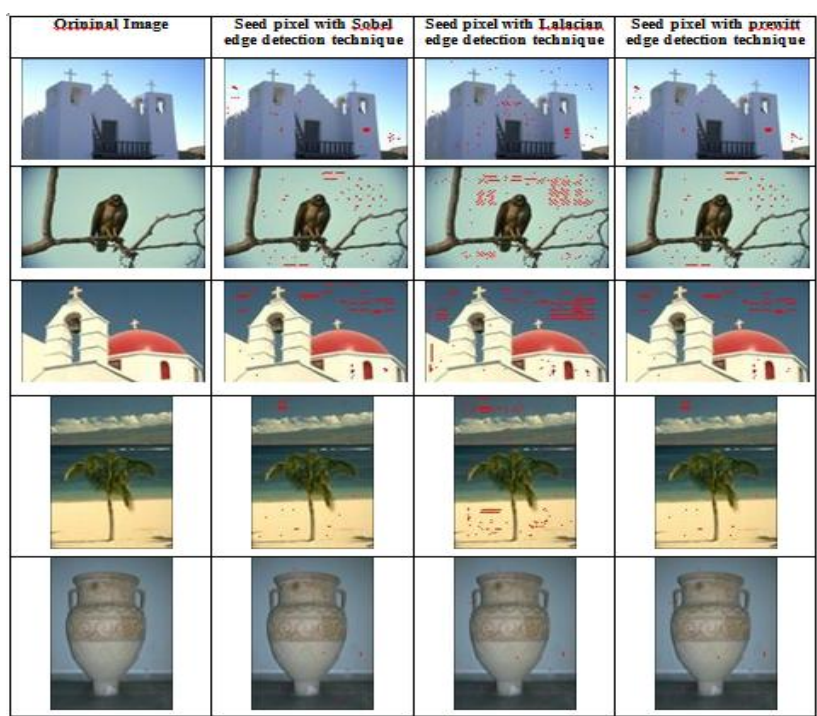

Figure 3: Proposed technique with sobel, laplacian and prewitt edge detection technique showing seed pixel in red color in different images. 
The position of seed pixels are shown in graphs for comparative analysis. In graphs, author uses different colors and markers to show seed pixels obtained with edge detection technique. Seed pixels in images with sobel, laplacian and prewitt are analyzed. The analysis based on the location of seed pixels show that seed pixels obtained with sobel and prewitt have common in large numbers than any other combination of seed pixels obtained with edge detection technique. The performance analysis shows that gradient based edge detection technique provide better result than other edge detection technique.

\section{Seed pixel with Sobel edge detection technique}

Seed pixel with Laplacian edge detection technique

. . .......... Seed pixel with Prewitt edge detection technique

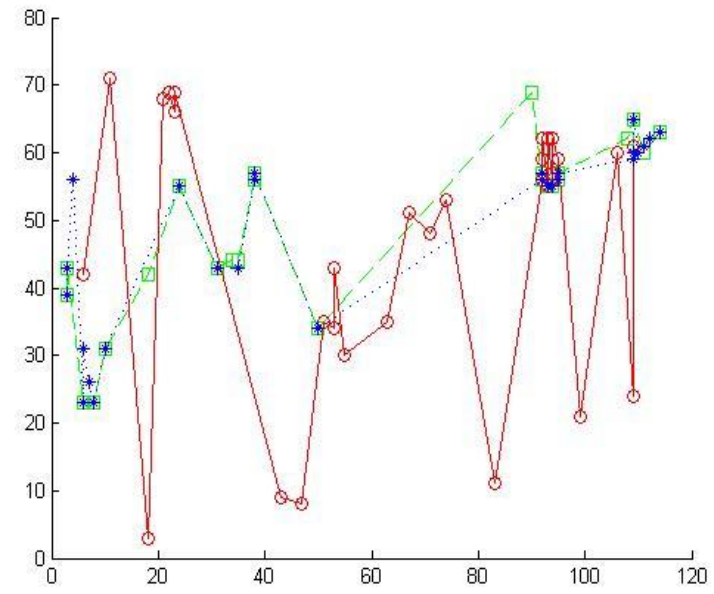

(a)

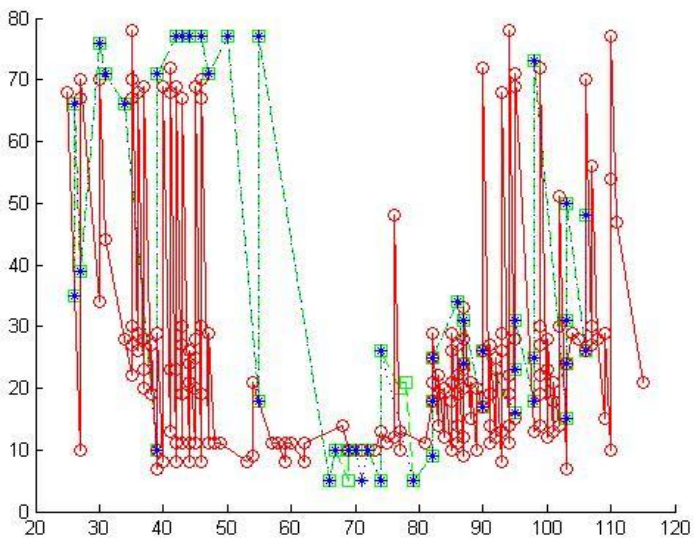

(b)

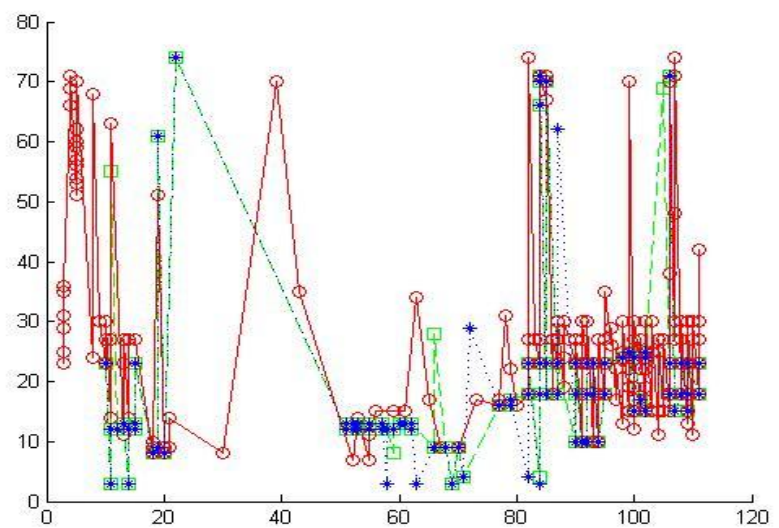

(c)

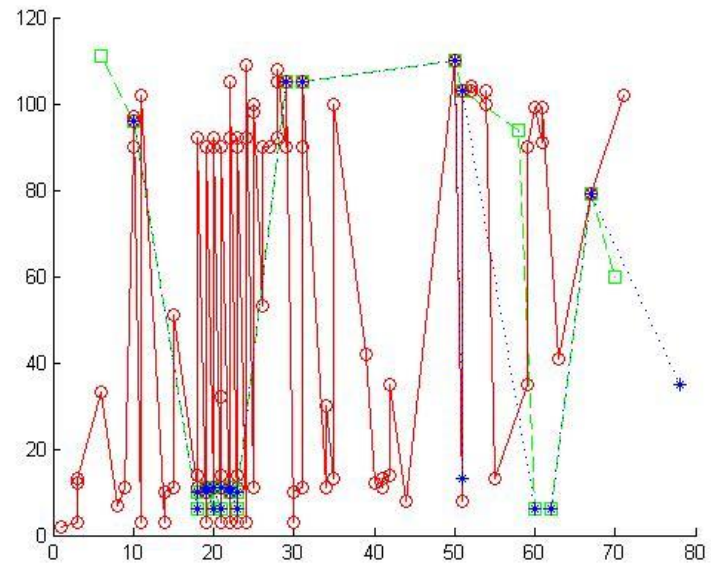

(d)

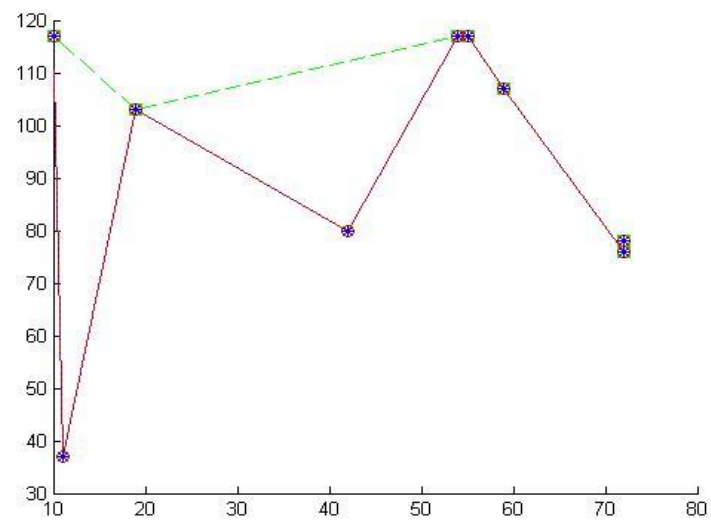

(e)

Figure 4: Position of seed pixels in graphs of images present in above table. Images are chosen in the same order as they present in table. 


\section{EXPERIMENTAL RESULTS}

The proposed technique has been implemented in MATLAB 7.5.0 in window 7. The performance parameter CPU: Intel(R) Core(TM) i3, $2.10 \mathrm{GHz}$, RAM memory: 4GB. The input images are color image obtained from Berkeley image segmentation dataset. Threshold value $(\Delta) 100$ is used in all results.

\section{CONCLUSION}

A new technique to select initial seed pixels has been proposed in this paper. This technique is based on edge detection technique. This technique uses two type of information: non-edge information and similarity behavior of pixels to its neighbors. The proposed technique is applied to different images with different edge detection technique and observed that gradient based edge detection technique such as sobel and prewitt provide better results.

\section{REFERENCE}

[1] Adams, R., Bischof, L., "Seeded region 1growing", IEEE Transactions on Pattern anlysis and Machine Intelligence, vol. 16, page 641-647, 1994.

[2] Om Prakash Verma, Madasu Hanmandlu, Seba Susan, Muralidhar Kulkarni and Puneet Kumar Jain "A Simple Single Seeded Region Growing Algorithm for Color Image Segmentation using Adaptive Thresholding" IEEE 978-0-7695-4437, 2011.

[3] Frank Y. Shih and Shouxian Cheng "Automatic seeded region growing for color image segmentation" Image and vision computing 23, 877-886, 2005.

[4] N. Chowdhury, B. Banerjee , T. Bhattacharjee "color image segmentation technique using "natural grouping" of pixels" 1985-2304, 2010.
[5] Gracia Guzzarria "Automatic Image Segmentation by Dynamic Region Growth and Multiresolution Merging “, 18-10, 2275 - 2288, 2009.

[6] A. V. Anjikar and V. K. Shandilya "Effective method of Initial Seed Selection Used for Color Image Segmentation" IJCTA, Vol 2 (3), 431-432.

[7] Thodeti Srikanth, Prof P. Pradeep Kumar, Ashwin Kumar "color image segmentation using watershed algorithm” IJCSIT, vol. 2 (5), 2332-2334,2011.

[8] Changmin Zhang, Shuaiqi Zhang, Junxia Wu, Shaoxiong Han "an improved watershed algorithm for color image segmentation" IEEE 978-0-7695-4647-6/12 2012.

[9] R. C. Gonzalez and R. E. Woods, digital image processing, Pearson education, 2002.

[10] Malik sikandar hayat khiyal, aihab khan, and amna bibi, "modified watershed algorithm for segmentation of 2D images" IISIT volume 6, 2009.

[11] Raman Maini, Dr. Himanshu Aggarwal, "Study and Comparison of Various Image Edge Detection Techniques" IJIP Volume (3): Issue (1).

[12] Mamta Juneja, Parvinder Singh Sandhu, "Performance Evaluation of Edge Detection Techniques for Images in Spatial Domain", 1793-8201, International Journal of Computer Theory and Engineering, Vol. 1, No. 5, December, 2009.

[13] Vineet Saini, Rajnish Garg, "A Comparative Analysis on Edge Detection Techniques Used in Image Processing”, IOSRJECE, 2278-2834 Volume 1, Issue 2, PP 56-59, 2012 